\title{
Splat Formation Mechanism in Thermal Spraying*
}

\author{
Masahiro FUKUMOTO**, Kun YANG**, Motohiro YAMADA** \\ and Toshiaki YASUI** \\ **Interface \& Surface Fabrication Laboratory, Toyohashi University of Technology, \\ 1-1, Tempaku-cho, Toyohashi, Aichi 441-8580, Japan \\ E-mail: fukumoto@tut.jp
}

\begin{abstract}
In order to understand the splat formation process of individual splat deposited by thermal spraying, commercially available Nickel powders with diameter of several tens micrometers were thermally sprayed onto mirror polished AISI304 substrate surface. The deposited splat shows transition phenomenon from splash type to disk one in flattening on collision onto substrate surface according to both substrate temperature and ambient pressure change. That is, transition temperature, $\mathrm{Tt}$ and transition pressure, Pt can be defined as critical values for the transitions. The three-dimensional map was set up to control the thermal spraying process. The observation on the bottom surface morphology on single splat indicates that the initial solidification may induce the splashing occurrence. Moreover, as the simulation of the real thermal spraying process, free falling experiment was conducted in this study. The thermal history of the free falling $\mathrm{Cu}$ droplet onto AISI304 substrate was investigated, indicating the flattening pattern is decided just after collision onto solid surface, which is enough earlier to finalization of the flattening.
\end{abstract}

Key words: Thermal Spraying, Flattening, Disk-Shaped Splat, Splash Splat, Transition, Heat Transfer, Initial Solidification

\section{Introduction}

Up to today, more and more mechanical equipment has been used in severer environments owing to the continuing development of industry and material science. However, using the materials with high performances to produce the product is always uneconomical, and it is impossible in some cases with the prevailing material processing technology. Since most of the material damage and failure start from the surface of the material, surface technology as a surface protection method by surface modification or cover with other materials to the substrate has been developed recently. Thermal spraying is such a method in which melted materials with high velocity are sprayed onto a surface to form a dense coating with advanced properties. This technique has been widely used in many industrial applications: mechanics, aeronautics, aerospace, chemistry and oil, electronic, military, automotive, medical, marine, and mining. Their development has continuously increased over the last decade, due to the high deposition rate and cost effective as compared to the other coating processes ${ }^{(1),(2)}$. The process reliability, however, has not been always established due to its lower controllability. As a flattening of an individual thermal sprayed particle on the substrate is a unit cell for the coating formation process, coating microstructure and corresponding properties, such as porosity and adhesion

*Received 28 June, 2011 (No. 11-0356) [DOI: 10.1299/jmmp.5.1001]

Copyright $\odot 2011$ by JSME 
strength, depend strongly on the flattening nature of each splat ${ }^{(3)}$. Therefore, it is necessary to study in detail the basic process of flattening behavior of the sprayed particles, not only for scientific interest, but also has technical consequences.

As reported up to now, effect of dominating process factors on the flattening behavior of an individual splat onto flat substrate surface has been systematically investigated through theoretical ${ }^{(4),(5)}$, numerical ${ }^{(6)-(8)}$ and experimental methods ${ }^{(9)-(11)}$ in the last few decades. However, the splat formation process of the thermal sprayed particles is not fully understood yet, therefore, there is a need for a deeper study of this aspect.

Generally, nearly all the previous studies observed the final splats without knowing the actual impact process, owing to the thermal spraying is a complex and short-period process, hereby, it is difficult to clarify the flattening and solidification behavior of the thermal sprayed particles directly with the prevailing technology. To overcome this difficulty, a free-falling experiment was carried out as a simulation of the thermal spray process, using both experiments and numerical simulation ${ }^{(12)-(15)}$. As the thermal spraying and free falling can be have a similar Re and Pe numbers, an analogy exist for both phenomena.

In this study, the flattening behaviors of individual thermal sprayed Nickel particle collected on the flat AISI304 substrate with various temperatures and/or under reduced ambient pressures were investigated systematically. The thermal history of millimeter-sized free falling Copper droplets during the spreading process onto the flat substrate was recorded as well. This article focuses, in particular, on the splat formation mechanism relating to transition based on the observation on single splat.

\section{Experimental procedure}

Thermal sprayed particles were collected on the substrates using atmospheric pressure spraying (APS) and low-pressure plasma spraying (LPPS) ${ }^{(16),(17)}$. Commercially available Ni powders with $53 \mu \mathrm{m}$ or less in diameter as shown in Fig. 1 (Kojundo Chemical Lab. Co., Ltd., Japan.) were used as the feedstock for the spraying. AISI304 plates with dimensions of $20 \mathrm{~mm} \times 20 \mathrm{~mm} \times 6 \mathrm{~mm}$ finally polished with $0.3 \mu \mathrm{m} \mathrm{Al}_{2} \mathrm{O}_{3}$ buff prior to spraying were used as substrates. The number of the particles deposited on the substrate in one passing of the shutter was around one hundred or more. In-flight temperature and velocity of the particles sprayed in atmospheric pressure was measured by DPV-2000 system.

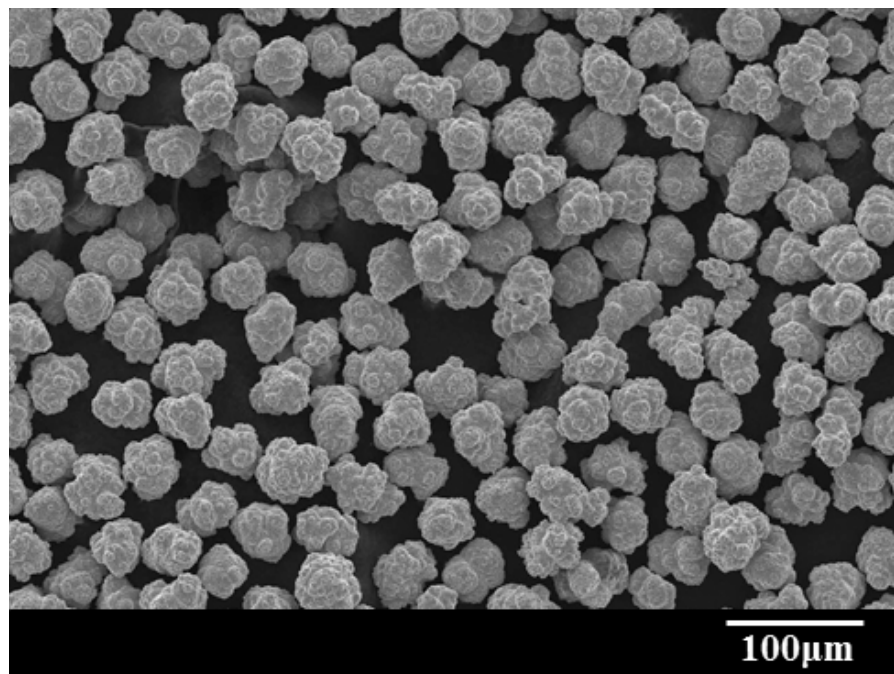

Fig. 1 SEM image of Ni powder used

The splat shape was evaluated using optical microscope (OM). Splats were classified as either disk-shaped splat or splash one, respectively. The number of disk-shaped splats and 
splash splats were counted on every trial in the experiments. The fraction of disk-shaped splat was defined as the ratio of the number of disk-shaped splats to the total splats. The splat morphologies in detail were observed by scanning electron microscope (SEM). First of all, the top surface morphologies of the splat collected under the designated conditions were observed. Following this, carbon tape was pressed on the sprayed region, and then pulled off, some splats were removed. The bottom surface of the splat collected on carbon tape was investigated with SEM ${ }^{(18)}$. In particular, the grain distribution at the splat bottom surface was examined using scanning ion beam (SIM). The shear adhesion strength of the fabricated coatings was evaluated by Autography AGS-L ${ }^{(19)}$.

The free falling experiments were conducted as shown in Fig. 2. As the melting point of Nickel is outside the measuring range of thermocouple, instead, commercially available $\mathrm{Cu}$ wire with a diameter of $2 \mathrm{~mm}(99.9 \%$ pure) was used as droplet material. It was melted by radio-frequency heating equipment prior to the falling. No.2000 waterproof paper polished AISI304 plate with $30 \mathrm{~mm} \times 30 \mathrm{~mm} \times 5 \mathrm{~mm}$ was used as substrate. To clarify the effect of substrate temperature and ambient pressure on heat transfer at interface between molten droplet and substrate surface, the thermal history of the droplet on the substrate was measured during the flattening and solidification process. As thermocouple junction can be connected by molten metal of the splat, the temperature in splat bottom part was recorded since droplet impinged onto substrate surface. The measuring method can be referred in our previous report ${ }^{(20)}$.

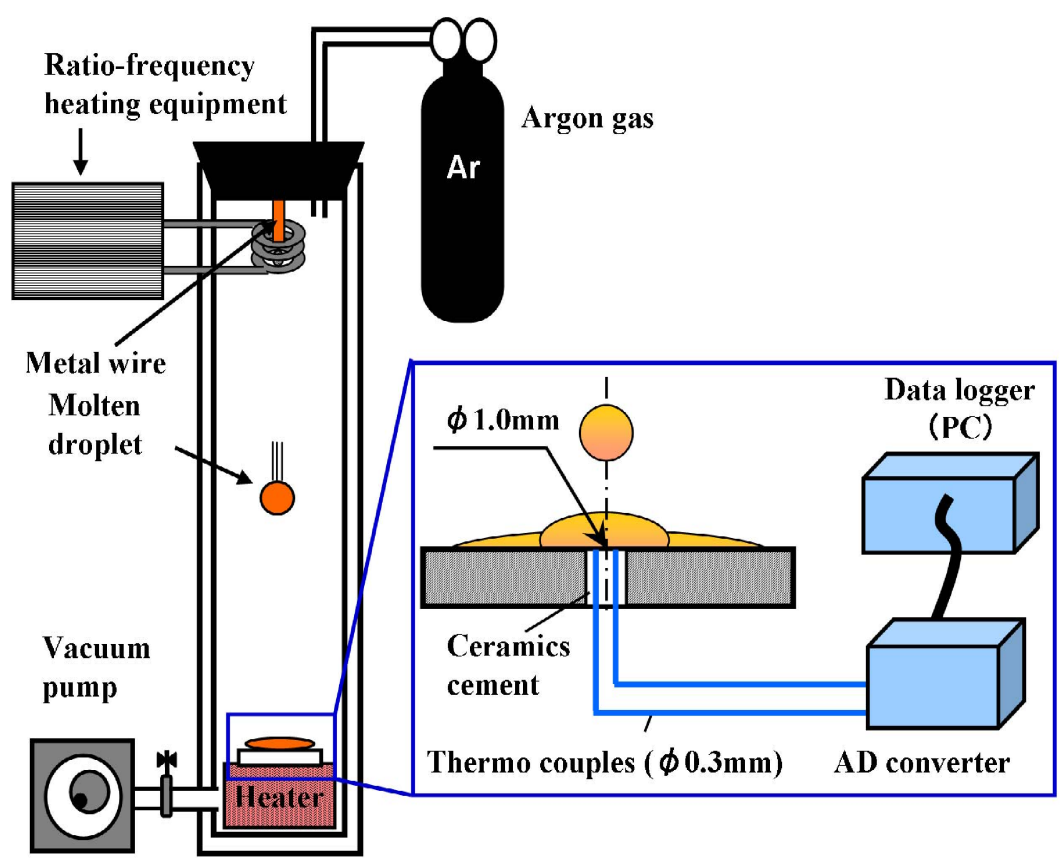

Fig. 2 Schematic of free falling experimental apparatus and temperature data logging system

\section{Results and discussion}

\subsection{Transition behavior of thermal sprayed particles}

Figure 3 shows the dependence both of the splat shape and coating adhesion strength on substrate temperature when Ni powders were thermally sprayed onto AISI304 substrate. According to the figure, a transition behavior of the deposited splat shape can be recognized, the splat on the substrate with room temperature performed as a splash shaped, ring shaped splash fingers were deposited around the central solidification area, the splat shapes undergoes a transition from a distorted shape with splash to a disk-shaped splat with the 
increase of substrate temperature, the central solidification area was enlarged, while few projections along the periphery of the splat can be observed. Actually, most of the feedstock materials sprayed onto the flat substrate shows such a transition behavior through increasing substrate temperature ${ }^{(21)-(24)}$. The transition temperature, $T t$, was defined as the critical temperature above which half of the splat was disk shaped. Meanwhile, the adhesion strength of coating fabricated under the same process as the splat collections increased drastically with the increase of substrate temperature. Its dependence on substrate temperature corresponds quite well to that of the splat shape on a flat substrate, in other words, the splat flattening nature likely has a strong influence on the coating characterizations.

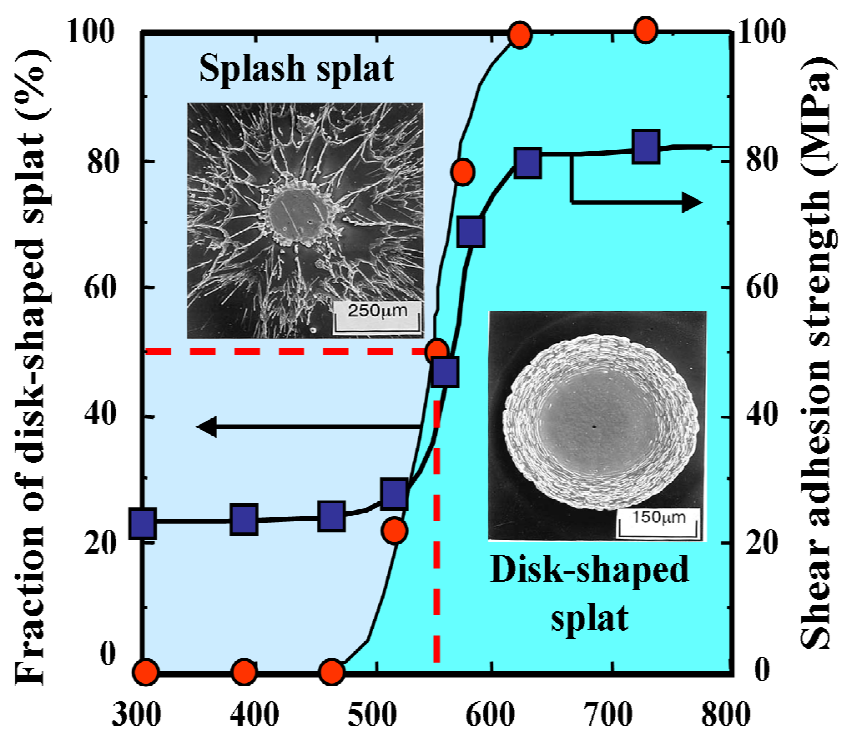

\section{Substrate temperature $(\mathrm{K})$}

Fig. 3 Dependence both of fraction of disk-shaped splat and adhesion strength on substrate temperature of Ni powders sprayed onto AISI304 substrate

Similarly, the Ni powders were thermally sprayed onto flat AISI304 substrate under various ambient pressures as well, while keep the substrate at room temperature during the splat collection. The splat collected on the flat substrate was distinguished as disk-shaped splat or splash one. Only the ratio between the area of splash finger and whole splat above around $50 \%$ was treated as splash one, the splat with only few short fingers connected with the central solidification zone was still treated as disk-shaped splat. All the splats deposited under different ambient pressures were distinguished with the same standard. It is clearly recognized that the splat pattern changed from the form with splashing to the one without splashing with the decrease of ambient pressure as shown in Fig. 4. While the ambient pressure was at atmospheric pressure, the splash region not connects to the central solidification area can be found, which was named as ring-shaped splash splat. With the continuing decrease of ambient pressure, the splat performs a uniform disk-like shape, which is formed by the continuous and stable flow from the impingement center of the particle. Similar transition phenomenon was report by other researchers ${ }^{(25),(26)}$. Accordingly, the transition pressure, $P t$, was defined as the critical pressure at which the fraction of disk-shaped splat exceeded $50 \%$ with reducing the ambient pressure.

Generally, splat shape on the flat substrate had a transitional changing tendency from a splash splat to a disk one was obtained with the increase of substrate temperature and/or the decrease of ambient pressure. Namely, both substrate temperature and ambient pressure may have an equivalent effect on the transition, the disk-shaped splat deposited instead of 
splashing on the flat substrate, which attribute to the favorable wetting of substrate by molten droplet generated either through removing the adsorbates on substrate surface ${ }^{(16),(17)}$, or surface roughness increase in nano-scale through substrate heating ${ }^{(27),(28)}$.

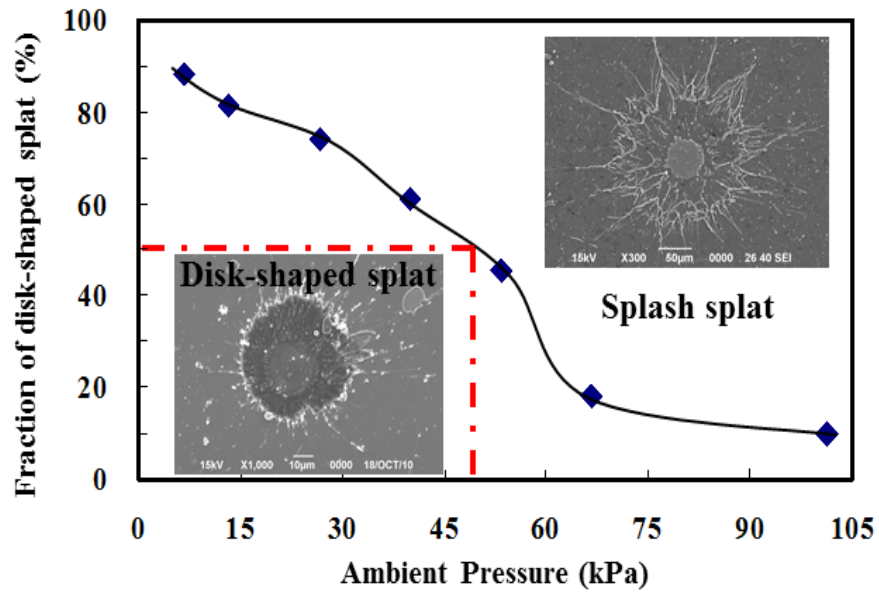

Fig. 4 Dependence of fraction of disk-shaped splat on ambient pressure of Ni powders sprayed onto AISI304 substrate

The dependence of fraction of disk-shaped splat both on the substrate temperature and ambient pressure in the deposition chamber was summarized schematically as shown in Fig. 5 , as a three-dimensional transition curvature. Therefore, by selecting the optimum conditions both in substrate temperature and ambient pressure in the thermal spraying process, we can design the coating microstructure and properties. Hence, thermal spray process can be controlled more effectively.

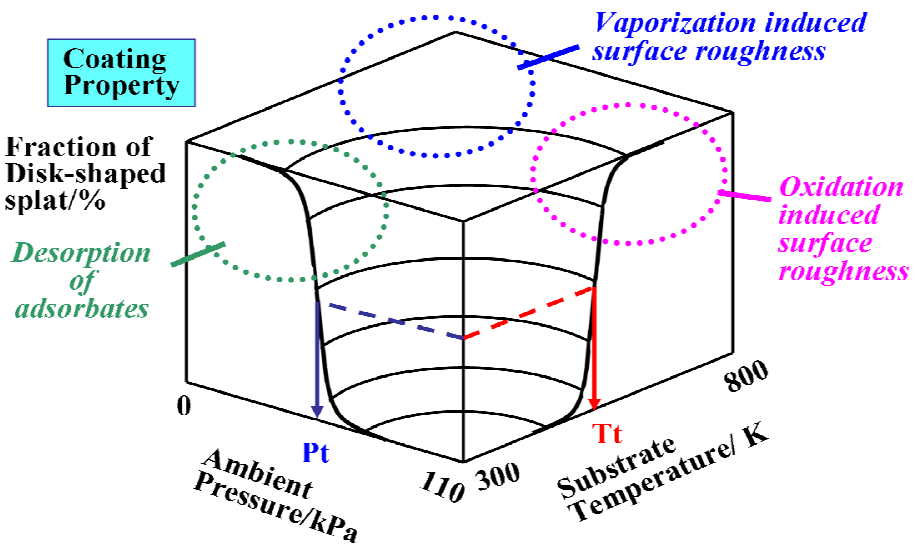

Fig. 5 Three dimensional map for control of thermal spray process

As a confirmation this hypothesis, three-dimensional transition curvature for the sprayed Ni particles onto AISI304 substrate were measured based on the experimental data as shown in Fig. 6. According to the figure, the equivalent effect of substrate temperature and ambient pressure on the transition behavior is recognized. Good adhesion strength and the corresponding properties for the fabricated coatings can be obtained both by substrate preheating and ambient pressure reduction in the thermal spraying. 


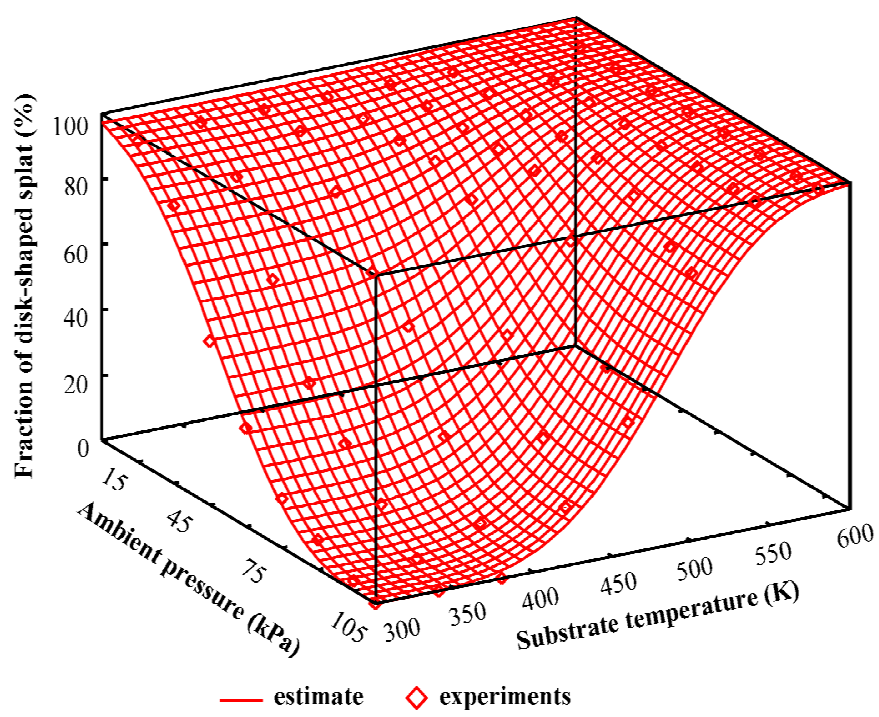

Fig. 6 Experimental results on three dimensional transition curvature of $\mathrm{Ni}$ powder sprayed onto AISI304 substrate

To summarize, there was no doubt about the transition behavior from splash type to disk-shaped one during thermal spraying process, but we still cannot answer why and how does disk-shaped splat deposited instead of splash one through substrate preheating and ambient pressure reduction. Aiming at answering the problem, the splashing number, K, based on the study of water and ethanol droplet, has been often mentioned recently ${ }^{(29)}$, which was described as

$\begin{array}{ll}\mathrm{K}=\mathrm{We}^{1 / 2} \mathrm{Re}^{1 / 4} & (\text { Eq } 1) \\ \mathrm{We}=\rho d v^{2} / \gamma & (\text { Eq 2) } \\ \mathrm{Re}=\rho \mathrm{\rho dv} / \eta & (\text { Eq 3) }\end{array}$

Here We and Re are the Weber and Reynolds number, and $\rho$ : density, d: diameter, $v$ : velocity, $\gamma$ : viscosity, $\eta$ : surface tension, respectively.

If $\mathrm{K}<3$, splat rebounds;

While $3<\mathrm{K}<57.7$, it results in deposition;

And, if $\mathrm{K}>57.7$, it induces splashing.

Therefore, the value of 57.7, was defined as the critical value as Kc. However, the K value was precisely calculated on the thermal sprayed $\mathrm{Ni}$ particles, through measuring the particle temperature and velocity using DPV-2000 system in this study. By introducing the measured temperature and velocity into each physical constant, such as density, viscosity and surface tension have been calculated by the following equations ${ }^{(30)}$ :

$$
\begin{aligned}
& \rho=\rho_{m p}-\rho_{0}(T-m p) \\
& \eta=\eta_{0} e^{-E / R T} \\
& \gamma=\gamma_{0}-\gamma_{m p}(T-m p)
\end{aligned}
$$


Here, mp means the melting point of feedstock powder, $\mathrm{E}$ is the activation energy and $\mathrm{R}$ is the gas constant. Accordingly, $\mathrm{K}$ values obtained under designated conditions were shown as shown in Fig. 7.

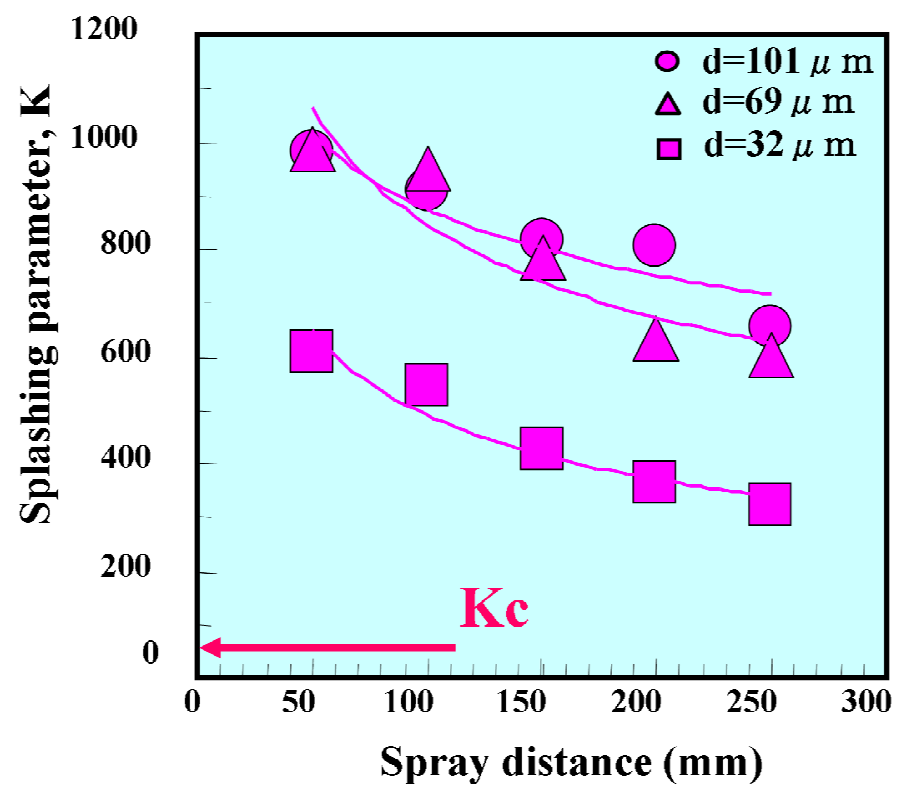

Fig. 7 Splashing parameter K of Ni powders sprayed onto AISI304 substrate

According to the result, larger $\mathrm{K}$ can be obtained when coarser feedstock materials were thermally sprayed onto flat substrate, while the $\mathrm{K}$ value decreases with the increase of the spray distance. However, these $\mathrm{K}$ values are still significantly larger than that of $\mathrm{Kc}$, regardless of the particle size, or the spray distance. Namely, regular sprayed particle has enough driving force for the splashing, but the disk-shaped splat was always appears on the hot substrate, or the lowered ambient pressure. C. Escure et al. proposed that, on the other hand, significant splashing occurred even the $\mathrm{K}$ is smaller than the critical value of 57.7 when alumina particles were thermally sprayed onto the stainless steel substrate ${ }^{(31)}$. In other words, for thermal spraying conditions involved in particle solidification, these critical values seem not applicable. Some other factors may have a stronger effect on the splat formation process, we need to verify the splat formation mechanism in more detail.

\subsection{Flattening and solidification behavior of free falling droplets}

Because the actual thermal spraying is a short period but complex process, feedstock powders with diameter of micrometer order were sprayed onto the flat substrate surface. It is difficult to measure the flattening and solidification process with the prevailing technology. To overcome this problem, millimeter-sized $\mathrm{Cu}$ droplets were free fallen onto flat AISI304 substrate through controlling substrate temperature and ambient pressure. The morphologies of droplet deposited under designated conditions were illustrated as Fig. 8. According to the results, the droplet collected on the substrate with room temperature at atmospheric pressure performed as splash shape, sharper and longer splash fingers can be found around the central solidification zone, the splash finger can be connected or separated with the central zone, however, with the increase of substrate temperature or the decrease of ambient pressure, the droplet shape changed transitionally from the pattern of splash to disk one, the splash fingers become shorter and smooth, which has a similar transition tendency as the real thermal spraying process. Accordingly, the investigation on the flattening behavior of thermal sprayed particles through observation on the free falling droplet should 
be reasonable and meaningful, regardless of the droplet temperature and velocity.

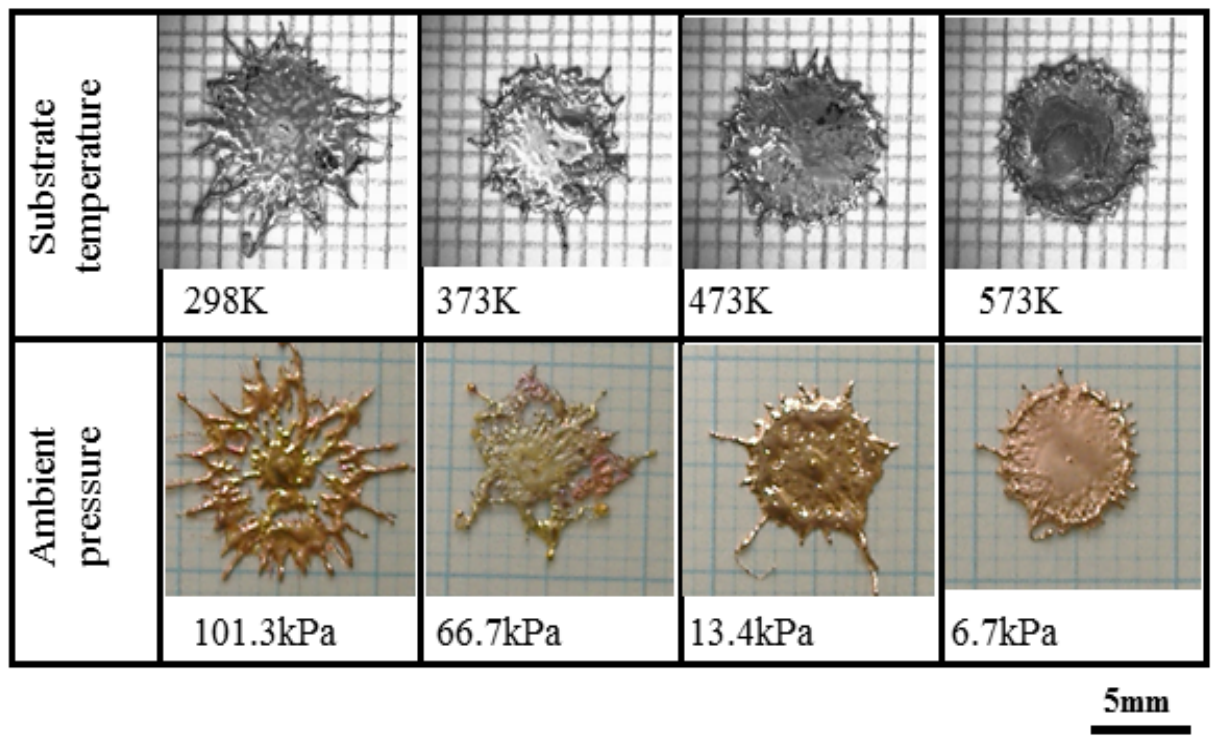

Fig. 8 Dependence of the shape of free falling $\mathrm{Cu}$ droplet deposited on AISI304 substrate on substrate temperature and ambient pressure

The temperature histories at the droplet-substrate interface of the splats deposited onto substrate kept at various temperatures are summarized in Fig. 9. All the measurements were conducted at atmospheric pressure, and substrate temperatures were room temperature, $423 \mathrm{~K}$ and $523 \mathrm{~K}$, respectively. Temperature elevation was recognized at first stage of splat impingement at all substrate heating conditions, indicating that the temperature of outside shell of the splat was lower than its inside. In the next stage, splat cooling started with splat flattening. Splat temperature elevation occurred again only on the substrate kept at 523K. This elevated temperature period can be estimated as a plateau in the solidification. The plateau appeared at $1000 \mathrm{~K}$ in this case, that is, it was slightly lower than the melting point of the droplet material. It means that droplet was super cooling condition when impinged onto the substrate. As the slope of the splat temperature history curve, $d T / d t$, was defined to be the cooling rate of the splat, the splat cooling rates on heated substrates were remarkable higher than those substrates with room temperature, and increases with substrate heating temperature. Similar result has been proposed while some kinds of micrometer-sized particles were thermally sprayed onto flat substrate surfaces ${ }^{(32)-(34)}$, which proposed that the investigation on the thermal history of the millimeter-sized free falling droplet is meaningful for understanding the individual thermal sprayed particle.

Moreover, the measurement of temperature histories at droplet-substrate interface of $\mathrm{Cu}$ splats deposited onto AISI304 substrate kept at various ambient pressures in deposition chamber are shown in Fig. 10. All the measurements were carried out on non-heated substrate, and ambient pressure was $101.3 \mathrm{kPa}, 40.0 \mathrm{kPa}$ and $6.7 \mathrm{kPa}$, respectively. According to the figure, similar cooling and solidification process was found through controlling the ambient pressure, while the cooling rate in the splat increases with decrease of ambient pressure. 


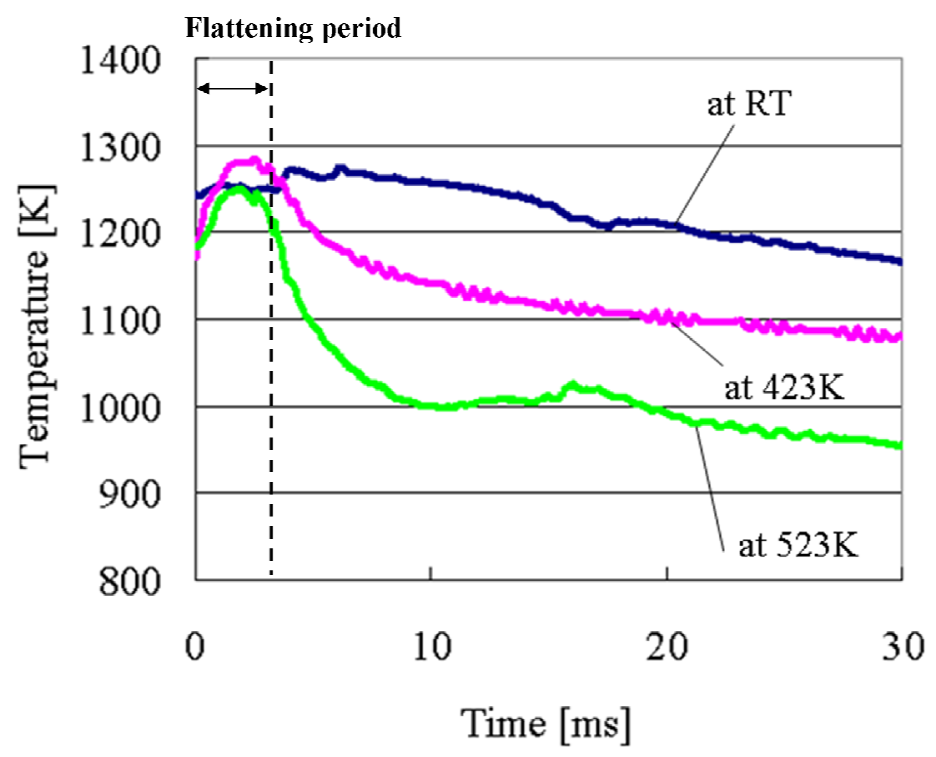

Fig. 9 Effect of substrate temperature on temperature history of free falling $\mathrm{Cu}$ droplet

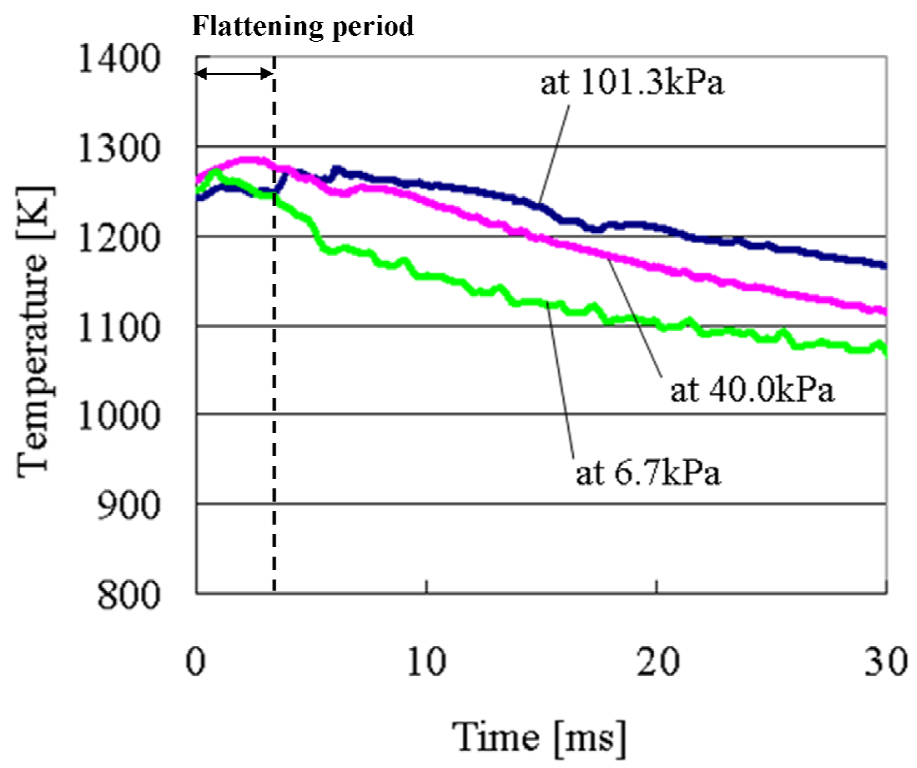

Fig. 10 Effect of ambient pressure on temperature history of free falling $\mathrm{Cu}$ droplet

To summarize, equivalent effect on the heat transfer character can be given both by substrate temperature and ambient pressure. The cause of the lower cooling rate on the substrate at room temperature and under atmospheric pressure is probably the adsorbates and condensates. Good contact, along with the favorable wetting of substrate by molten droplet can be obtained by removing the adsorbed/gas condensation through substrate heating or ambient pressure reduction ${ }^{(16),(17)}$, thereby, the heat transfer between the molten droplet and substrate surface was enhanced, corresponding with higher cooling rate, and finally affect the splat formation.

However, the flatting process is a very short period after the droplet impact onto the substrate surface in all the experimental processes as indicated in Fig. 9 and 10, that is, only few milliseconds were spent prior to the solidification process. Actually, the time scale of thermal sprayed particle is much shorter than that of free falling droplet, however, the experimental conditions was designated as the equivalent Reynolds numbers and Peclet numbers with each other. Consequently, the thermal sprayed particle might have similar 
flattening and solidification behavior with free falling droplet. This indicates that flattening pattern is decided so quickly, namely, just after collision onto solid surface, which is enough earlier to finalization of the flattening rather than the lateral solidification process on the substrate surface. Hence, the mechanism on this quick decision has to be clarified.

\subsection{Initial solidification induced splashing model}

In order to obtain a deep understanding of the splat flattening and solidification process onto flat substrate surface, the bottom surface morphologies of Nickel splats collected under various ambient pressures were investigated as illustrated in Fig. 11. From the figure, when the splat was collected under atmospheric pressure (Fig. 11a), numerous nano-pores and complicated structure was observed on its bottom surface. On the central collision zone, indicated as mark 1, some adsorbed gas induced pores can be found. Outside the central zone was marked with $\mathbf{2}$, relatively dense structure was observed. The result indicates that relatively slower cooling occurred in this zone and it may be brought about higher heat flux from the splat. Also, the desorbed condensates or trapped gas form the periphery where the molten droplet meet the substrate surface cannot enter into the bottom surface of the splat. Therefore, the zone without any pores was created. Ring shaped porous zone marked as $\mathbf{3}$ was observed outside of this central zone. The pores in this zone may be generated by the rapid cooling without eliminating the adsorbed condensations at the interface during the flattening process. Actually, on the outside periphery zone marked as 4, irregular pores and splash fingers can be found in the splat.

Some splats were collected under reduced pressure, it was found that almost no pore can be observed at the bottom surface of the splat deposited under $6.7 \mathrm{kPa}$. The bottom surface looks quite dense and homogeneous (Fig. 11b), which indicates more intimate contact can be expected at the lower pressure conditions. The most common condensate is water from moisture. Desorption tends to occur when the substrate temperature rises or ambient pressure decreases. Actually, often molecules form multi-layers, and some are adsorbed on already adsorbed molecules. BET isotherm ${ }^{(35)}$ suggests that lack of a true chemical bond between adsorbed gas molecular and substrate except the first layer, so that the physical adsorption can be removed easily by reducing the ambient pressure. As the dynamic impact pressure of the droplet towards the substrate surface can be very high and concentrates at a small contacting area ${ }^{(36)}$, the contact of the droplet at an early stage of deformation is favorable.

Similar changing behavior in bottom surface microstructure was recognized in some other metallic splats deposited by atmospheric plasma spraying (APS) through controlling the substrate temperature.

Following this, the grain distribution at bottom surface of splat deposited under different pressures was examined using scanning ion beam (SIM) as illustrated in Fig. 12. From the figure, coarser grain can be found at the central collision zone of splat deposited under atmospheric pressure (Fig. 12a). With the increase of splat spreading direction, ring-shaped porous zone marked as $\mathbf{3}$ with smaller grain size was observed outside of this central zone. This might be the as defined initial solidified zone., Once the molten droplet impacted onto the flat substrate, there was continuous heat flux from flattening droplet to cold substrate, in particular at the initial impinging zone, due to the dynamic impact pressure and intimate contact. However, this impact pressure spread quickly with the droplet flattening onto substrate surface, therefore the heat flow was weakened along with the flattening direction, the temperature at the bottom surface of the splat decreased rapidly after impinging. As a result, most of the initial solidification might occur at the periphery of the splat in a very short period. While almost no initial solidification occur at the initial central impinging zone, because of the continuous heat flux from the molten droplet, which result in the formation of coarser grain This initial solidified layer might become the trigger of the lateral 
rapid flattening fluid and restrain the heat transfer from the molten droplet to substrate, then induced the occurrence of splashing.
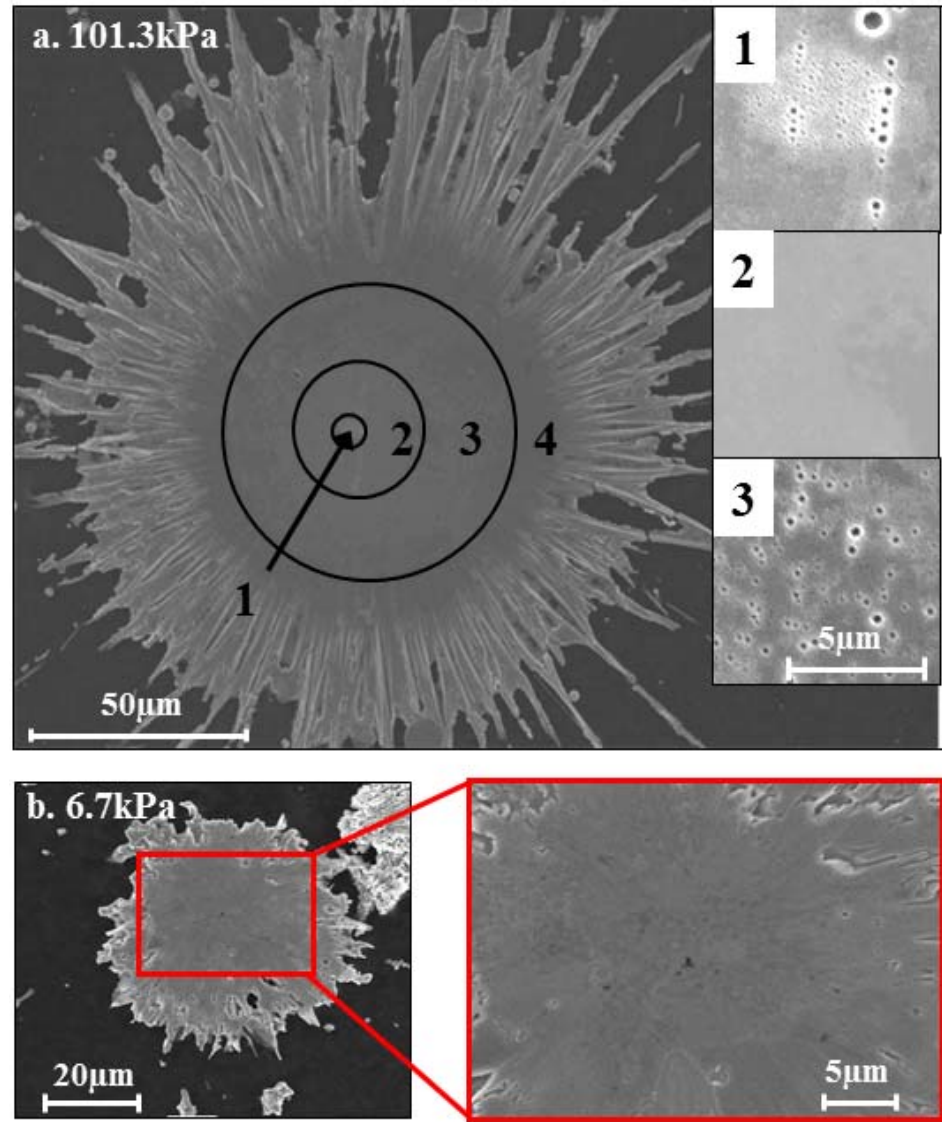

Fig. 11 Bottom surface morphology of Ni splats deposited onto AISI304 substrate under various ambient pressures

a. $101.3 \mathrm{kPa}$

Center

Periphery

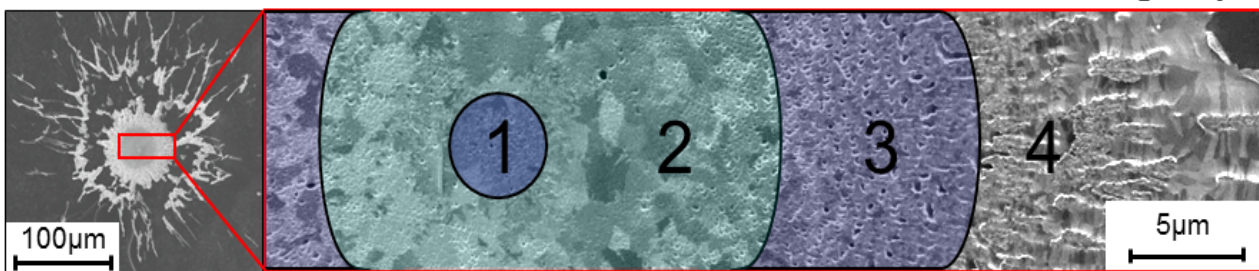

\section{b. $6.7 \mathrm{kPa}$}

\section{Center}

Periphery

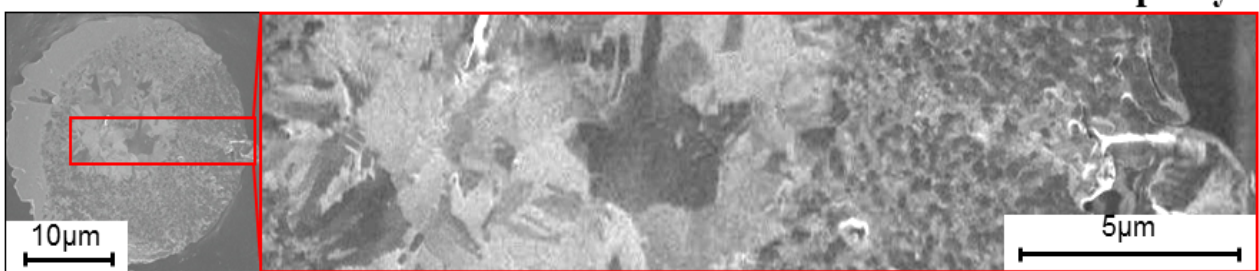

Fig. 12 Grain distribution at bottom surface of Ni splats deposited onto AISI304 substrate under various ambient pressures

On the other hand, coarser grain size can be observed at the central zone when the spraying was conducted under reduced ambient pressure (Fig. 12b), while the grain size decreases gradually far away from the central collision zone, only few fine grains were found at the periphery of the splat, in other words, the degree of initial solidification occurred at this zone during the splat flattening process is not so strong. With the decrease 
of ambient pressure, most of the adsorbed gas/condensation on substrate surface was removed, and more favorable wetting of substrate by molten droplet can be obtained. As a result, once the molten droplet impact onto the flat substrate surface, the interface temperature increase much faster than that of the splat deposited under high pressure condition, consequently, the initial solidified zone was significantly smaller than that obtained under atmospheric pressure, only a thin initial solidified layer might form at the periphery of the splat, the thickness of this layer might much thinner than that of the splat deposited under high pressure conditions, this thin layer could prevent the motion of flattening boundary, and finally promote the formation of disk-shape splat.

On the basis of experimental result, the initial solidification occurred at splat bottom surface once the molten droplet impact on the flat substrate likely affect the splat formation process. However, intensive investigation is needed to clarify the influence in more detail.

\section{Conclusions}

Aiming at clarifying the splat formation mechanism of thermal sprayed particles, commercially available Ni powders were thermally sprayed onto hot substrate and/or under lowered ambient pressures. The flattening and solidification behavior of the individual splat was systematically investigated. Thermal history of free falling $\mathrm{Cu}$ droplet onto flat substrate under designated conditions was recorded as well. The results obtained in this study are summarized as follows:

(1) Transition behavior of splat shape from a splash splat to a disk one was recognized both by increasing substrate temperature and reducing ambient pressure. The critical transition substrate temperature and ambient pressure was defined as transition temperature $(T t)$, and transition pressure (Pt.), respectively. By selecting the optimum operating conditions both in substrate temperature and ambient pressure, thermal spray process can be controlled effectively.

(2) Substrate temperature and ambient pressure have equivalent effect on heat transfer between droplet and substrate. The final splat shape was decided just after collision onto solid surface, namely, just after collision onto solid surface, which is enough earlier to finalization of the flattening rather than the lateral solidification process on the substrate surface.

(3) The ring shaped initial solidification surrounding the central dense zone at the bottom surface was found, in particular, under high pressure condition. This initial solidified layer of the splat may become the trigger of the lateral flattening droplet, which might induce the splashing occurrence.

\section{Acknowledgement}

The authors would like to acknowledge Mr. K. Tanaka, Y. Ebisuno, S. Yoshida, and D. Mano for their assistance of the experiments. The research was supported both by the Grant-in-Aid for Scientific Research of the Ministry of Education, Science, Culture and Sports in Japan and by a special research fund in Toyohashi University of technology.

\section{References}

(1) M. L. Thorpe, Advanced Materials \& Processes, Vol. 143, No. 5 (1993), pp. 50-61.

(2) P. Fauchais, M. Fukumoto, A. Vardelle, and M. Vardelle, Journal of Thermal Spray Technology, Vol. 13, No. 3 (2004), pp. 337-360. 
(3) M. Fukumoto, H. Hayashi, and T. Yokoyama, Journal of Japan Thermal Spraying Society, Vol. 32, No. 3 (1995), pp. 149-156. (in Japanese)

(4) J. Madjeski, International Journal of Heat and Mass Transfer, Vol. 19 (1976), pp. 1009-1013.

(5) H. Fukanuma, Journal of Thermal Spray Technology, Vol. 3, No. 1 (1994), pp. 33-44.

(6) R. Dhiman, A. McDonald, and S. Chandra, Surface and Coatings Technology, Vol. 201 (2007), pp. 7789-7801.

(7) S. Chandra, and P. Fauchais, Journal of Thermal Spray Technology, Vol. 18, No. 2 (2009), pp. 148-180.

(8) A. T. T. Tran, S. Brossard, M. M. Hyland, B. J. James, and P. Munroe, Plasma Chemistry and Plasma Processing, Vol. 29 (2009), pp. 475-495.

(9) C. Moreau, P. Gougeon, and M. Lamontagne, Journal of Thermal Spray Technology, Vol. 4, No. 1 (1995), pp. 25-33.

(10) V. Pershin, M. Lufitha, S. Chandra, and J. Mostaghimi, Journal of Thermal Spray Technology, Vol. 12, No. 3 (2003), pp. 370-376.

(11) A. McDonald, C. Moreau, and S. Chandra, Surface and Coatings Technology, Vol. 202 (2007), pp. 23-33

(12) Y. Heichal, and S. Chandra, Journal of Heat Transfer, Vol. 127, No. 11 (2005), pp. 1269-1275.

(13) M. Pasandideh-Fard, S. Chandra, and J. Mostaghimi, International Journal of Heat and Mass Transfer, Vol. 45 (2002), pp. 2229-2242.

(14) S. D. Aziz, and S. Chandra, International Journal of Heat and Mass Transfer, Vol. 43 (2000), pp. 2841-2857.

(15) R. G. Azar, Z. Yang, S. Chandra, and J. Mostaghimi, International Journal of Heat and Fluid Flow, Vol. 26, No. 2 (2005), pp. 334-347.

(16) K. Yang, K. Tomita, M. Fukumoto, M. Yamada, and T. Yasui, Journal of Thermal Spray Technology, Vol. 18, No. 4 (2009), pp. 510-518.

(17) K. Yang, M. Fukumoto, T. Yasui, and M. Yamada, Journal of Thermal Spray Technology, Vol. 19, No. 6 (2010), pp. 1195-1205.

(18) M. Qu, and A. Gouldstone, Journal of Thermal Spray Technology, Vol. 17, No. 4 (2008), pp. 486-494.

(19) M. Fukumoto, M. Mashiko, M. Yamada, and E. Yamaguchi, Journal of Thermal Spray Technology, Vol. 19, No. 1-2 (2010), pp. 89-94.

(20) M. Fukumoto, K. Yang, K. Tanaka, T. Usami, T. Yasui, and M. Yamada, Journal of Thermal Spray Technology, Vol. 20, No. 1-2 (2011), pp. 48-58.

(21) M. Vardelle, A. Vardelle, A.C. Leger, P. Fauchais, and D. Gobin, Journal of Thermal Spray Technology, Vol. 4, No. 1 (1995), pp. 50-58.

(22) L. Bianchi, A. Grimaud, F. Blein, P. Lucchese, and P. Fauchais, Journal of Thermal Spray Technology, Vol. 4, No. 1 (1995), pp. 59-66.

(23) M. Fukumoto, H. Nagai, and T. Yasui, Journal of Thermal Spray Technology, Vol. 15, No. 4 (2006), pp. 759-764.

(24) M. Fukumoto, T. Yamaguchi, M. Yamada, and T. Yasui, Journal of Thermal Spray Technology, Vol. 16, No. 5-6 (2007), pp. 905-912.

(25) M. F. Morks, Y. Tsunekawa, M. Okumiya, and M.A. Shoeib, Journal of Thermal Spray Technology, Vol. 12, No. 2 (2003), pp. 282-289.

(26) S. Sampath, and H. Herman, Journal of Thermal Spray Technology, Vol. 5, No. 4 (1996), pp. 445-456.

(27) R. N. Wenzel, Industrial \& Engineering Chemistry, Vol. 28, No. 8 (1936), pp. 988-994.

(28) T. Uelzen, and J. Muller, Thin Solid Films, Vol. 434 (2003), pp. 311-315.

(29) C. Mundo, M. Sommerfeld, and C. Tropea. International Journal of Multiphase Flow, Vol. 21, No. 2 (1995), pp. 151-173. 
(30) Metals Reference Book, $5^{\text {th }}$ ed., Butterworths, 1976.

(31) C. Escure, M. Vardelle, A. Vardelle, and P. Fauchais, Visualization on the impact of drops on a Substrate in Plasma Spraying: Deposition and Splashing Modes, Proceedings of the International Thermal Spraying Conference 2001, (2001), pp. 805-812.

(32) A. McDonald, C. Moreau, and S. Chandra, Surface and Coatings Technology, Vol. 202 (2007), pp. 23-33.

(33) A. McDonald, M. Lamontagne, C. Moreau, and S. Chandra, Thin Solid Films, Vol. 514 (2006), pp. 212-222.

(34) A. McDonald, C. Moreau, and S. Chandra, International Journal of Heat and Mass Transfer, Vol. 50 (2007), pp. 1737-1749.

(35) S. Brunauer, P. H. Emmett, and E. Teller, Journal of American Chemical Society, Vol. 60, No. 2 (1938), pp. 309-319.

(36) C. J. Li, and J. L. Li, Journal of Thermal Spray Technology, Vol. 13, No. 2 (2004), pp. 229-238. 